\title{
Cigarras (Hemiptera: Cicadidae: Tibicinidae) Associadas ao Cafeeiro: Distribuição, Hospedeiros e Chave para as Espécies
}

\author{
Nilza M. Martinelli ${ }^{1}$ e Roberto A. Zucchi ${ }^{2}$ \\ ${ }^{1}$ Departamento de Defesa Fitossanitária, FCAV/LJNESP, 14870-000, Jaboticabal,SP. \\ ${ }^{2}$ Departamento de Entomologia, ESALQ/USP, Caixa postal 9, 13418-900, Piracicaba,SP.
}

An. Soc. Entomol. Brasil 26(1): 133-143 (1997)

Cicadas (Hemiptera: Cicadidae: Tibicinidae) on Coffee Plants:

Distribution, Hosts and Key to Species

\begin{abstract}
The geographical distribution of 10 species of cicadas associated to coffee (Coffea arabica) plants, in Minas Gerais, São Paulo e Paraná States (Brazil) is reported. Informations on geographical distribution of these species of cicadas in the New World, except Brazil, were based on the existing literature. For Brazil, the occurrence of species were based on the literature survey, on entomological collections and on specimens captured. Seven other hosts, besides coffee were recorded: sponge tree (Acacia farnesiana), false brazilian wood (Caesalpinia peltophoroides), Cassia spp., mulberry tree (Morus alba), avocado pear tree (Persea americana), angico (Piptadenia sp.) and cação tree (Theobroma cacao). Identification keys based on external morphology and on male genitalia are presented.
\end{abstract}

KEY WORDS: Insecta, cicada, hosts, survey, identification.

RESUMO - É apresentada a distribuição de 10 espécies de cigarras associadas ao cafeeiro, Coffea arabica, nos Estados de Minas Gerais, São Paulo e Paraná, Brasil. A informação sobre a distribuição geográfica destas espécies no Continente Americano, afora o Brasil, foi baseada na literatura. Para o Brasil, as localidades de ocorrência de cigarras foram baseadas na literatura, nos levantamentos realizados e em exemplares de coleções nacionais. Outros sete hospedeiros, além do cafeeiro, foram também registrados: esponjeira (Acacia farnesiana), sibipiruna (Caesalpinia peltophoroides), Cassia spp., amoreira (Morus alba), abacateiro (Persea americana), angico (Piptadenia sp.) e cacaueiro (Theobroma cacao). São apresentadas chaves de identificação, baseadas nas características gerais e na genitália masculina.

PALAVRAS-CHAVE: Insecta, cigarras, hospedeiros, levantamento, identificação.

Nos últimos anos, ao se tomarem pragas importantes do cafeeiro (Coffea arabica), o interesse sobre as cigarras aumentou consideravelmente. São escassos os conhecimentos sobre as cigarras do Brasil e sua biologia é pouco conhecida, mesmo para as espécies de importância agrícola. Apenas recentemente foram realizados estudos taxonômicos sobre as cigarras-do-cafeeiro (Martinelli 1985).

As cigarras-do-cafeeiro, no Brasil, estão registradas para os Estados de Minas Gerais, São Paulo e Paraná, onde têm causados sérios 
problemas à cultura (Metcalf 1963, Fonseca \& Araújo 1939, Martinelli 1985). Entretanto, a distribuição dessas cigarras não está restrita apenas às regiões produtoras de café. Dessa forma, apresentam uma distribuição muito ampla, pois o cafeeiro não é o único recurso trófico das cigarras, muito embora os hospedeiros de cigarras sejam poucos conhecidos. No entanto, os surtos atuais de cigarras em cafeeiros estão diretamente associados a destruição de seus hospedeiros nativos, principalmente nos cerrados, para o cultivo de cafezais.

Os trabalhos relacionados com as espécies de cigarras-do-cafeeiro, com informações sobre distribuição geográfica e hospedeiros, foram conduzidos por Jacobi (1907), Hempel (1913), Goding (1925), Fonseca \& Araújo (1939), Costa Lima (1942), Davis (1944), Schiottfeldt (1944), Fonseca (1945), Torres (1945), Metcalf (1963), Heinrich \& Pupin Neto (1964), Silva et al. (1968), Ruffínelli (1970) e Duffels \& Van der Laan (1985).

As chaves dicotômicas para gêneros, que ocorrem na Argentina, permitindo identificar Quesada, Fidicina e Dorisiana foram apresentadas por Déletang $(1919,1923)$. A literatura nacional carece de pesquisas referentes à classificação das espécies de cigarras. Apenas recentemente, a taxonomia das cigarrasdo-cafeeiro foi revisada por Martinelli \& Zucchi (1987,1989abc).

Neste trabalho é apresentada a distribuição geográfica, os hospedeiros e chaves de identificação para 10 espécies de cigarras associadas ao cafeeiro.

\section{Material e Métodos}

As cigarras foram coletadas utilizando-se uma armadilha constituída de uma armação de ferro coberta com tela de nailon, colocada sob a copa do café. Procederam-se também as coletas manuais ou através de rede entomológica. Após a coleta, os exemplares foram mortos e conservados em frascos etiquetados contendo álcool a $70 \%$ e posteriormente, montados e etiquetados.
Para o estudo da genitália, separou-se o abdomen com auxílio de pinça, submetendoo ao aquecimento em banho-maria, durante 15 a 30 minutos, em solução de $\mathrm{KOH}$ a $10 \%$. Em seguida o material foi lavado em água, colocado em fenol por 24 horas e conservado em tubos com glicerina, devidamente etiquetados. A terminologia da genitália do macho foi baseada nos trabalhos de Hayashi (1974, 1975, 1976) e Duffels (1982). As sinonímias das espécies estudadas constam do catálogo de Metcalf (1963) e Duffels \& Van Der Laan (1985) sendo assim omitidas neste trabalho. As figuras foram confeccionadas com auxílio de uma câmara clara acoplada no microscópio estereoscópico.

As localidades de ocorrência de cigarras foram baseadas na literatura, nas coletas realizadas e em exemplares de coleções nacionais (Fazenda Experimental de São Sebastião do Paraíso-EPAMIG, Departamento de Entomologia - ESALQ/USP, Departamento de Defesa Fitossanitária-FCAVJ/UNESP, Seção de Entomologia-IAC e Museu de Zoologia-USP). A caracterização das espécies foi apresentada em trabalhos anteriores (Martinelli \& Zucchi 1987,1989 abc.).

\section{Resultados e Discussão}

Distribuição Geográfica. O conhecimento atual do padrão de distribuição das espécies de cigarras no Brasil é ainda muito incipiente, uma vez que os registros são baseados em coletas esporádicas. De um modo geral, os estudos sobre esses insetos têm sido negligenciados em todo o continente americano. Entretanto, a distribuição das cigarras-do-cafeeiro no Brasil foi apresentada recentemente por Martinelli (1990) e Oliveira \& Thomazzielo (1991).

De todas as espécies de cigarras estudadas, a mais amplamente distribuída é Quesada gigas (Oliv.) (Fig. 1). Essa espécie apresenta uma distribuição vasta, sendo a única espécie, entre as 10 estudadas, referida para a América do Norte e Antilhas (Metcalf 1963). No Brasil, 


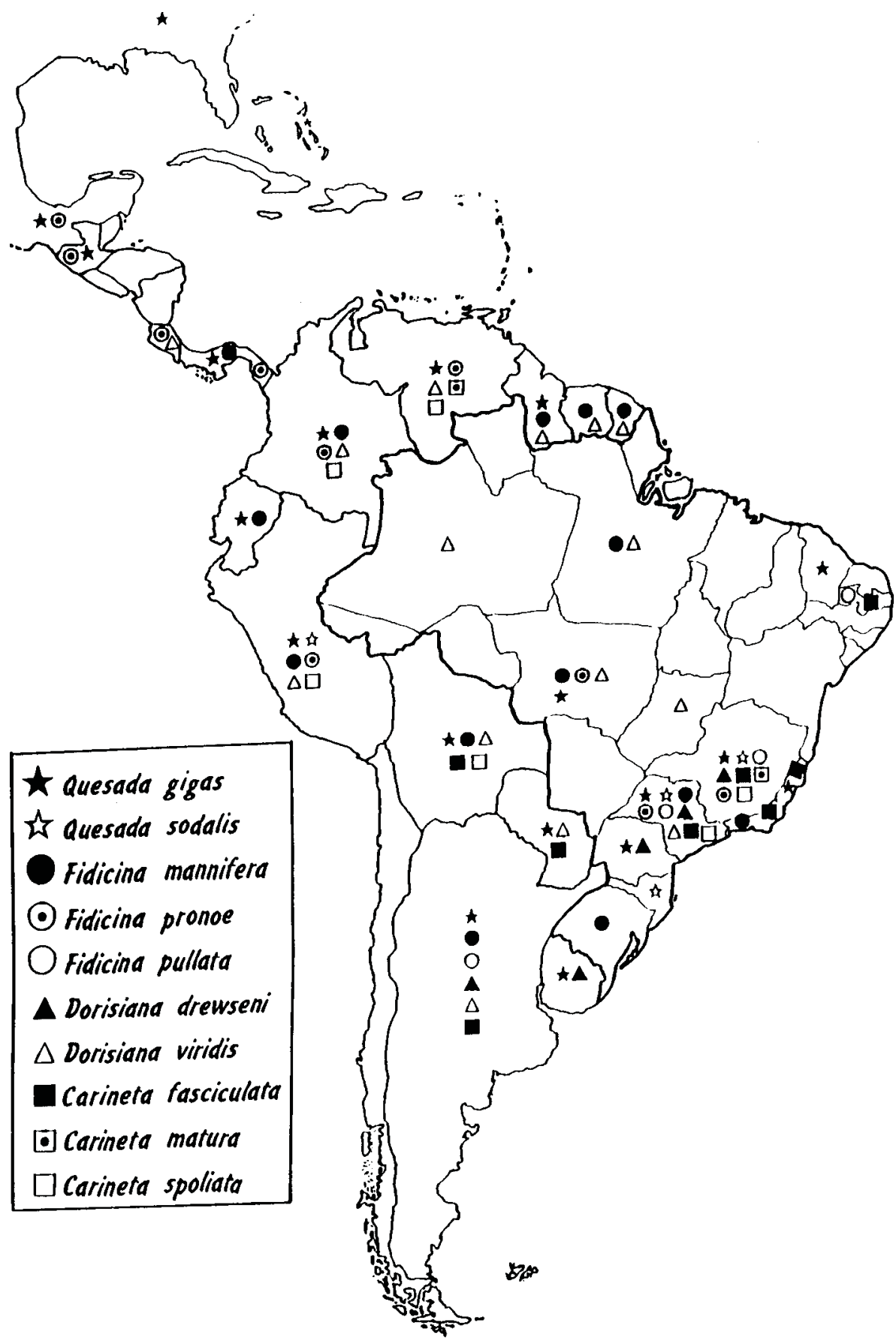

Figura 1. Distribuição geográfica das espécies de cigarras no Continente Americano. 
esta espécie encontra-se, quase que exclusivamente, nos principais Estados produtores de café (Minas Gerais, São Paulo e Paraná) (Martinelli 1985, Fonseca \& Araújo 1939,Heinrich 1967, Martinelli \& Zucchi 1987) e em Mato Grosso (Metcalf 1963). Quesada sodalis (Walk.) é referida em cafezais de Minas Gerais, São Paulo (Metcalf 1963 e Fonseca \& Araújo 1939) e Santa Catarina (Martinelli 1985). Entretanto, $Q$. sodalis não tem sido coletada atualmente em cafeeiros.

Com relação as espécies do gênero Fidicina, observa-se que $F$. pronoe (Walk.) e $F$. mannifera (Fabr.) ocorrem desde o México até a Argentina ( Metcalf 1963). No Brasil, a ocorrência de $F$. pronoe é verificada nos Estados de Mato Grosso e São Paulo (Metcalf 1963, Martinelli \& Zucchi 1989a). A ocorrência desta espécie também foi constatada em Minas Gerais. F. mannifera ocorre nos Estados do Pará, Mato Grosso, Rio de Janeiro, São
Paulo e Rio Grande do Sul (Distant 1881, Fonseca \& Araújo 1939, Metcalf 1963 ).F.pullata (Berg) é registrada apenas no Brasil, nos Estados de Paraíba, Minas Gerais e São Paulo (Silva et al. 1968, Metcalf 1963, Fonseca \& Araújo 1939). A primeira constatação de $F$. pronoe em cafezal foi observada no Estado de São Paulo (Martinelli \& Zucchi 1989a); aqui é referida pela primeira vez para o Estado de Minas Gerais. F. pullata e F. mannifera não tem sido observadas atualmente em cafeeiros.

Dorisiana drewseni (Stäl) tem sua ocorrência assinalada na Argentina, Uruguai (Metcalf 1963, Ruffínelli 1970) e Brasil, nos Estados de Minas Gerais, São Paulo e Paraná (Metcalf 1963, Martinelli \& Zucchi 1989b). Dorisiana viridis (Oliv.) está registrada também nos países limítrofes ao Brasil. Apresenta ampla distribuição no território nacional, ocorrendo nos Estados do Amazonas, Pará, Goiás, Mato Grosso e São Paulo (Metcalf 1963, Martinelli \& Zucchi 1989b).

18
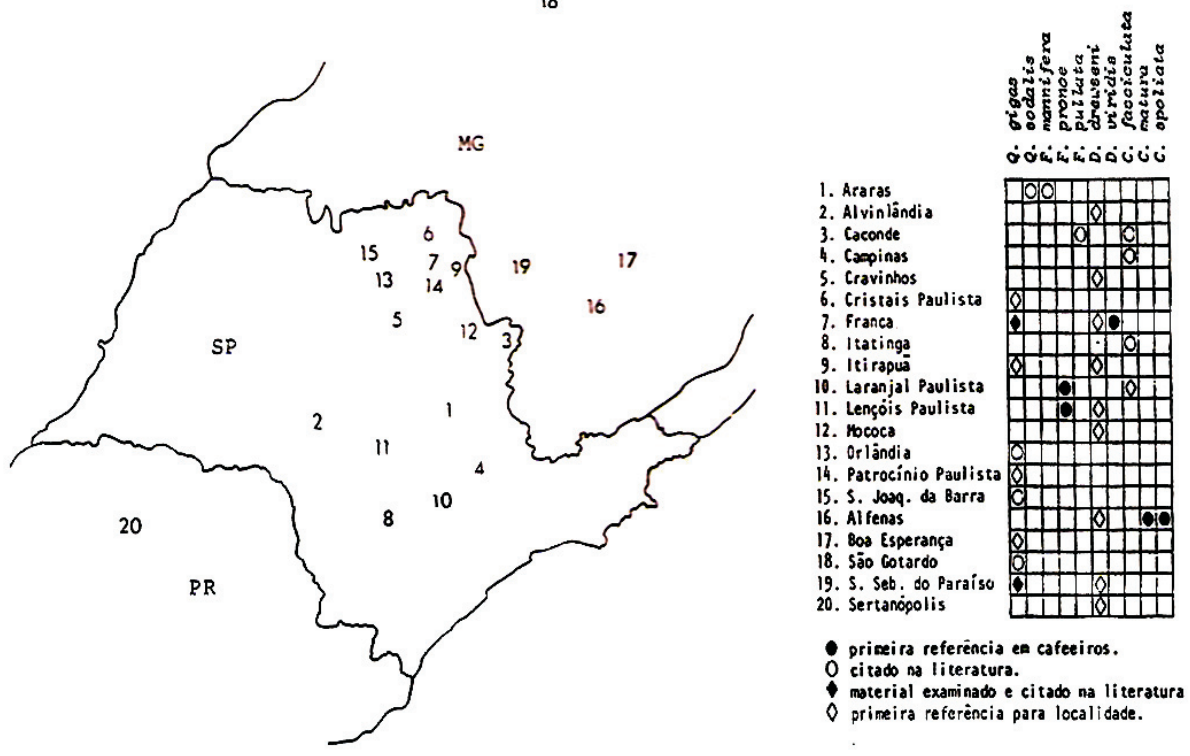

Figura 2. Distribuição das cigarras associadas ao cafeeiro em diferentes municípios dos Estados de Minas Gerais (MG), São Paulo (SP) e Paraná (PR). 


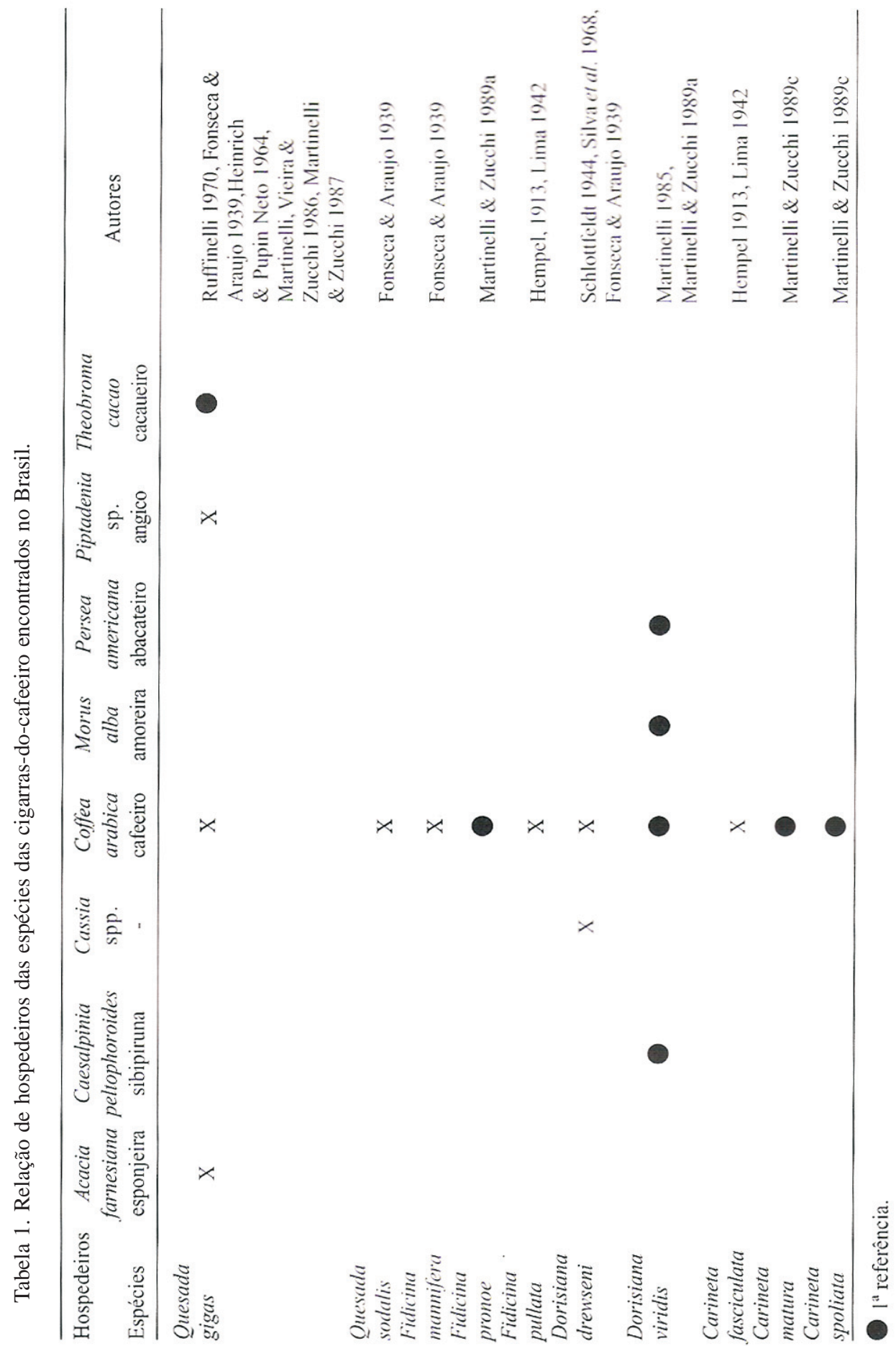


Dentre as espécies de Carineta, associadas ao cafeeiro, C. matura (Dist.) é de ocorrência restrita, pois além do Brasil, no Estado de Minas Gerais (Martinelli \& Zucchi 1989c), tem sido registrada na Venezuela (Metcalf 1963). Por outro lado, as outras duas espécies de Carineta apresentam distribuição mais ampla. C.fasciculata (Germ.) é encontrada na Bolívia, Paraguai e Argentina (Metcalf 1963, Jacobi 1907) e no Brasil (Paraíba, Minas Gerais, Espirito Santo, Rio de Janeiro e São Paulo) (Metcalf 1963 .Silva et al. 1968, Martinelli \& Zucchi 1989c), enquanto C. spoliata (Walk.) apresenta distribuição mais ampla, pois além da Bolívia é registrada na Colômbia, Peru e Venezuela (Metcalf 1963). No Brasil esta espécie é referida exclusivamente para os Estados de Minas Gerais e São Paulo (Martinelli \& Zucchi 1989c).

Há várias referências sobre ataques de cigarras em diferentes regiões dos Estados de Minas Gerais e São Paulo, nas quais as cigarras têm sido denominadas apenas em nível de gênero (Souza et al. 1983). Desta forma, foram consideradas, as identificações específicas como primeiro registro de ocorrência. Por outro lado, todas as ocorrências de cigarras para os Estados de Mato Grosso, baseadas na literatura, não permitem saber se os registros referem-se atualmente para esse Estado ou para Mato Grosso do Sul. Neste caso, os registros foram mantidos de acordo com os trabalhos originais, i. e., Q. gigas, F. mannifera, $F$. pronoe e D. viridis ocorrendo no Estado do Mato Grosso (Metcalf 1963).

Através de observações de campo, pôdese observar que a maior região cafeeira do Brasil (sul de Minas Gerais e Alta Mogiana em São Paulo), encontra-se altamente infesta-
Gerais, nove em São Paulo e uma no Paraná). Recentemente, quatro espécies ( $F$. pronoe, $D$. viridis, C. matura e C. spoliata) foram referidas também em cafeeiros (Martinelli 1985) (Fig.2).

Hospedeiros. Apenas para algumas espécies de cigarras são conhecidos seus hospedeiros (Silva et al. 1968). E pronoe, D. viridis, C. matura e C. spoliata foram constatadas infestando cafeeiros (Martinelli \& Zucchi 1989 $\mathrm{abc}$ ), sendo este o primeiro hospedeiro conhecido para essas quatro espécies. Entretanto, D. viridis foi observada em abacateiro (Persea americana), amoreira (Morus alba) e sibipiruna (Caesalpinia peltophoroides) (Martinelli 1985). Esta espécie e Q. gigas foram associadas a um maior número de hospedeiros (Tabela 1).

A maioria das espécies assinaladas em cafeeiros foi constatada, até o momento, apenas neste hospedeiro. Este fato denota que pouco é conhecido sobre os hospedeiros de cigarrras, pois seguramente estas espécies também ocorrem em outras plantas.

Nos primeiros relatos de ocorrência de cigarras em cafezais, $Q$. sodalis, $F$. mannifera e $F$. pullata eram mencionadas infestando o café. Entretanto, nos levantamentos realizados recentemente, não foram constatadas a presença das mesmas (Martinelli 1985). Atualmente, $Q$, gigas, $F$. pronoe, $D$. drewseni, $D$. viridis, C. fasciculata, C. matura e C. spoliata são as espécies associadas ao cafeeiro.

Identificação. Nas chaves apresentadas foram incluídas todas as espécies de cigarras associadas com o cafeeiro, independentemente se ocorrem ou não atualmente nesta cultura, (vide Martinelli \& Zucchi 1987, 1989abc para a caracterização das espécies). 

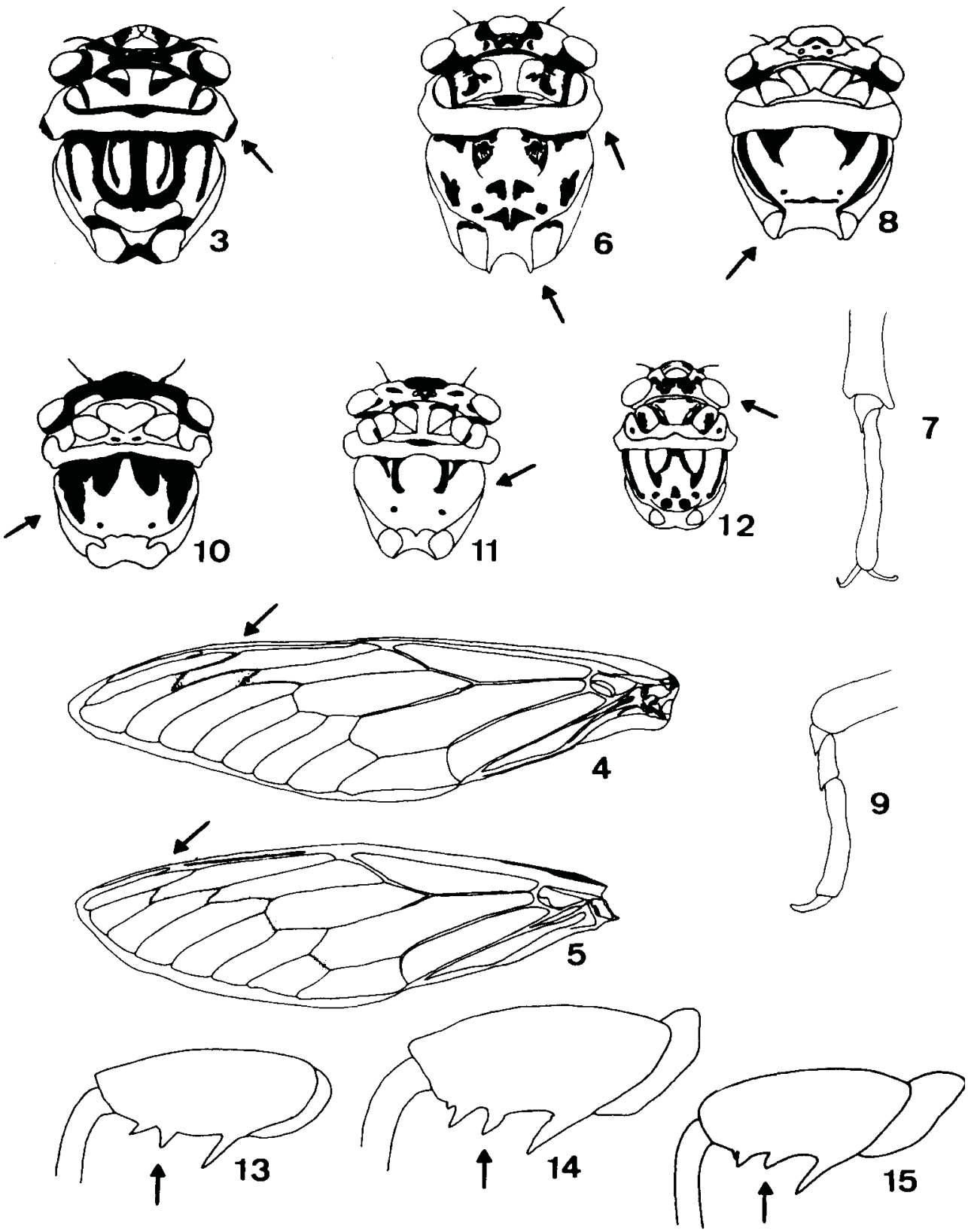

Prancha 1. Fig. 3 e 4 - Quesada gigas (pronoto; asa); Fig. 5 - Q. sodalis (asa); Fig. 6 e 7 - Fidicina mannifera (pronoto, tarso, elevação cruciforme); Fig. 8 - F. pronoe (elevação cruciforme); Fig. 9 e 10 - Dorisiana drewseni (tarso, mesonoto); Fig. 11 - D. viridis (mesonoto); Fig. 12 e 13 - Carineta matura (cabeça, fêmur anterior); Fig. 14 - C. fasciculata (fêmur anterior); Fig. 15 - C. spoliata (fêmur anterior). 

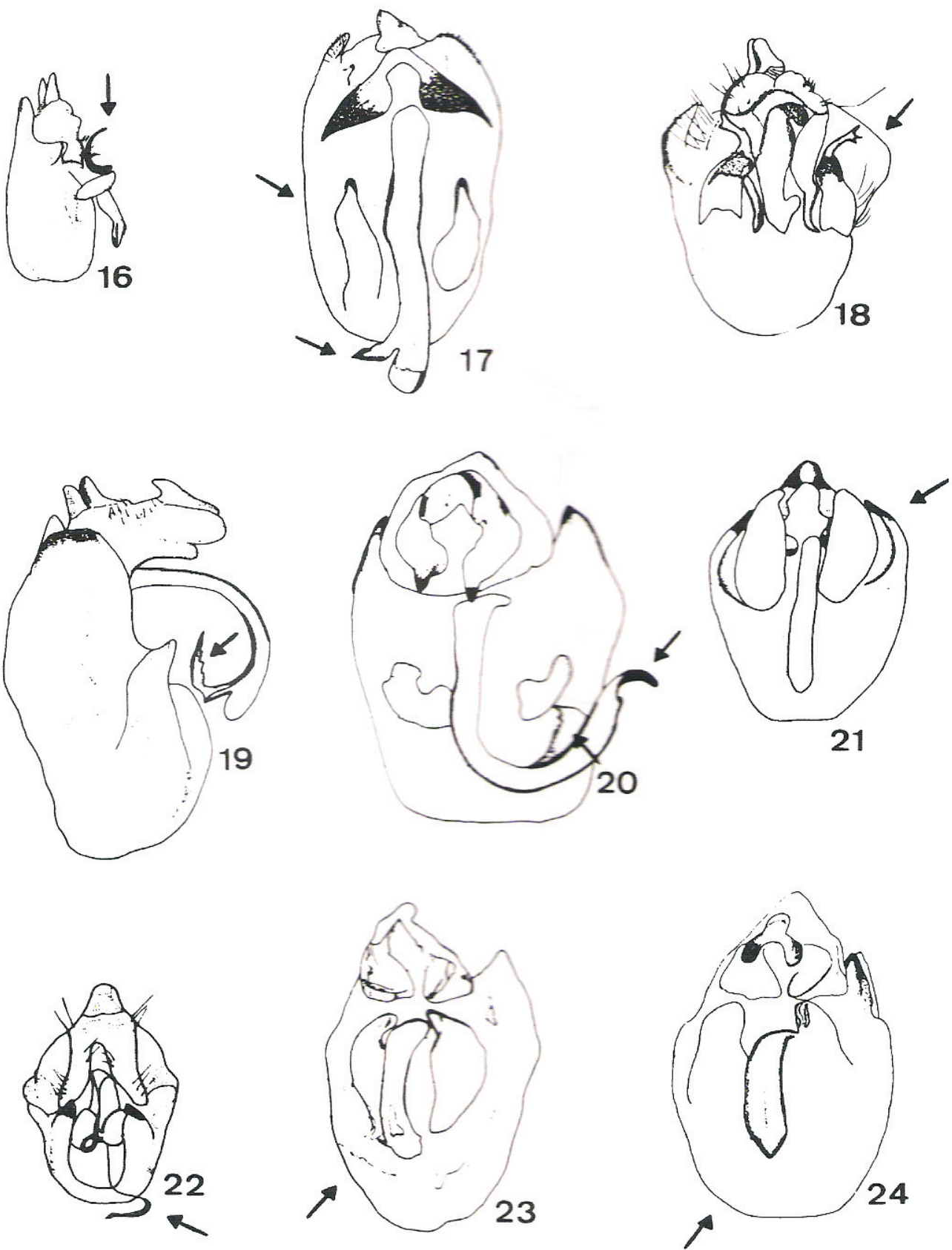

Prancha 2. Fig. 16 - Carineta matura (lobo basal do pigóforo - Ibp); Fig. 17 - Dorisiana viridis (Ibp, aedeagus); Fig. 18 - C. fasciculata (Ibp); Fig. 19 - Fidicina pronoe (aedeagus); Fig. 20 - F. mannifera (idem); Fig. 21 - D. drewseni (processo lateral do pigóforo); Fig. 22 C. spoliata (aedeagus); Fig. 23 - Quesada gigas (idem); Fig. 24 - Q. sodalis (idem). 


\section{Espécies de Cigarras Associadas ao Cafeeiro} (Machos e Fêmeas)

1. Ângulo posterior do pronoto dilatado (Fig. 3) Quesada

a. mancha escura na base da $2^{a}$ e $3^{\text {a }}$ células apicais.(Fig.4)..... Q. gigas $a^{\prime}$, mancha escura na base da $2^{a}$ a $5^{\text {a }}$ e $7^{\text {a }}$ células apicais (Fig.5) $Q$. sodalis

l'. Ângulo posterior do pronoto não dilatado (Fig. 6) .2

2. Tarsos dímeros( Fig. 7).......... Fidicina

a. coloração escura intensa .......F. pullata

a', coloração diferente. .. b

b. elevação cruciforme com as projeções não pontiagudas e afastadas entre si; nervuras transversais não manchadas. (Fig. 8) F. pronoe

b', elevação cruciforme com as projeções posteriores pontiagudas e próximas entre si; nervuras transversais manchadas (Fig. 6) .......... mannifera

2'. Tarsos trímeros (Fig. 9). 3

3. Cabeça (incluindo olhos) quase tão larga quanto a base do mesonoto. (Fig. 10) .Dorisiana

a. manchas do mesonoto largas, as laterais mais desenvolvidas que as internas (Fig. 10)......D. drewseni

a'. manchas do mesonoto estreitas, as laterais mais curtas que as internas (Fig 11). D. viridis

3'. Cabeça (incluindo olhos) mais estreita que a base do mesonoto (Fig. 12). Carineta

a. fêmur anterior com 3 espinhos (Fig. 13) C. matura

a', fêmur anterior com 4 espinhos b

b. segundo espinho voltado para o ápice do fêmur (Fig. 14) C. fasciculata

b', segundo espinho voltado para a base do fêmur (Fig. 15) C. spoliata
Espécies de Cigarras Associadas ao Cafeeiro (Genitália Masculina)

1. Lobo basal do pigóforo com prolongamento apical (Fig. 16) ......... 2

l'. Lobo basal do pigóforo sem prolongamento apical (Fig. 17) 3

2. Prolongamento do lobo basal do pigóforo quitinoso (Fig. 16).. C. matura

2'. Prolongamento do lobo basal do pigóforo com o ápice ramificado ou bífido

(Fig. 18) C. fasciculata

3. Aedeagus bífido (Fig. 17)................... 4

3'. Aedeagus simples (Fig. 20).................. 5

4. Porção bífida do aedeagus pontiaguda (Fig. 17)............ D . viridis

4'. Porção bífida do aedeagus com projeção denteada (Fig. 19) ...F. pronoe

5. Ápice do aedeagus com prolongamento quitinoso e projeção denteada pré-apical (Fig. 20) F. mannifera

5'. Ápice do aedeagus diferente

(Fig.21) 6

6. Processo lateral do pigóforo estreito, curvo e com o ápice quitinoso (Fig. 21)............. D. drewseni

6'. Processo lateral do pigóforo diferente 7

7. Aedeagus com um longo filamento apical (Fig. 22)...... C. spoliata

7'. Aedeagus sem filamento apical........... 8

8. Aedeagus com espinho pré-apical (Fig. 23) Q. gigas

8'. Aedeagus sem espinho pré-apical e ápice triangular em vista ventral (Fig. 24).... Q. sodalis

\section{Literatura Citada}

Costa Lima, A. 1942. Ordem Homoptera,p. 735 . In Insetos do Brasil. $3^{\circ}$ tomo. Rio de Janeiro, ENA, 327p. (Série Didática n. 4). 
Davis,W.T. 1944. The remarkable distribution of an American cicada; a new genus and other cicada notes. J. New York Entomol. Soc.52: 213-222.

Delétang. L. F. 1919. Contribución al estudio de los Cicádidos (Cicadidae) Argentinos (Hemiptera-Homoptera) ensayo filogenético. An. Soc. Cient. Argentina 88: 25-94.

Delétang, L. F. 1923. Monografia de los cicádidos (Cicadidae) Argentinos y relación de estos con la fauna sudamericana. An. Museo Nac. Hist. Nat. 31:538- 649.

Distant,W. L. 1881. Rhynchota: Homoptera - contributions to the knowledge of fauna and flora of México and Central America. Biol.Centr.Amer. 1: 1-16.

Duffels,J.P. 1982. Brachylobopyga decorata n.gen.,n.sp.from Sulawaesi, a new taxon of the subtribe Cosmopsaltriaria (Homoptera, Cicadoidea: Cicadidae). Ent. Ber. 42: 156-160.

Duffels, J. P. \& P. A. Van der Laan. 1985. Catalogue of the Cicadoidea (Homoptera, Auchenorhyncha 1956-1980. Amsterdam, Kluwer Academic Pulishers Group, 414p.

Fonseca, J. P. 1945. As cigarras do cafeeiro e seu combate. Bol. Agric. 8: 297- 304.

Fonseca, J. P. \& R. L.Araujo. 1939. Informações sobre a praga das cigarras em São Paulo e sobre as possibilidades de seu combate. Biológicos: 285-291.

Goding, F. W. 1925. Synopsis of the Cicadidae of Ecuador. Rev. Colegio Nac. Vicente Rocafuerte7:2-34.

Hayashi, M. 1974. The cicadas of the genus Platypleura (Homoptera, Cicadidae) in the
Rykuyu Archipelago, with the description of a new species. Kontyû 42: 232-235.

Hayashi, M. 1975. On the species of the genus Meimuna Distant (Homoptera, Cicadidae) of the Ryukyus. Kontyû 43:281-298.

Hayashi, M. 1976. On the species of the genus Moggania Amyot et Serville, 1843 (Homoptera, Cicadidae), of the Ryukyus and Taiwan. Kontyû 44:27-42.

Heinrich,W. 0.1967. Cicada- a coffee pest in Brasil. World Crops 19:43-47.

Heinrich.W. O. \& J. Pupin Neto. 1964. Experiências de campo para verificar a eficácia de alguns inseticidas sistémicos e de solo no combate às ninfas de cigarras (Homoptera, Cicadidae) em raízes de cafeeiro. Arq. Inst. Biol. 31:5-11.

Hempel, A. 1913. As cigarras do cafeeiro. Fazendeiro 6: 93-94.

Jacobi, A. 1907. Homoptera Andina. Die Zikaden des Kordillerengebietes von Sudamerikanach systematik und vertreitung. I Cicadidae. K. Zool. u. Anthrop.- Ethnog. Mus.Abhandl.u.Ber.11: 1-28.

Martinelli, N. M. 1985. Espécies de cigarras (Homoptera-Cicadidae) associadas ao cafeeiro. Tese de doutorado, ESALQ/USP, Piracicaba, 66p.

Martinelli, N. M. 1990. Café. As cigarras que causam danos à cultura. Correio Agrícola. 2:11-13.

Martinelli, N. M. \& R.A. Zucchi. 1987. Cigarras associadas ao cafeeiro. I. GêneroQuesada Distant, 1905 (Homoptera, Cicadidae, Cicadinae). An. Soc. Entomol. Brasil 16: 51-60. 
Martinelli, N. M. \& R.A. Zucchi. 1989a. Cigarras associadas ao cafeeiro. II. Gênero Fidicina Amyot \& Serville, 1843 (Homoptera, Cicadidae, Cicadinae). An. Soc. Entomol. Brasil 18:5-12.

Martinelli, N. M. \& R.A. Zlicchi. 1989b. Cigarras associadas ao cafeeiro. III. Gênero Dorisiana Metcalf, 1952 (Homoptera, Cicadidae, Cicadinae). An. Soc. Entomol. Brasil 18: 5-12.

Martinelli, N. M. \& R. A. Zucchi. 1989c. Cigarras associadas ao cafeeiro. TV Gênero Carineta A. \& S., 1843 (Homoptera, Tibicinidae, Tibicininae). An. Soc. Entomol. Brasil 18:13-22.

Martinelli, N.M., R.D. Vieira \& Zucchi. 1986. Descrição e ocorrência de Quesada gigas (Olivier, 1970) (Hom., Cicadidae) em cacaueiros no Estado de São Paulo. Cien. Agron.1:5-6.

Metcalf, Z.P. 1963. General Catalogue of the Homoptera. Cicadoidea. Parts 1 -2, Fase. VIU. Raleigh, Waverly Press, 919 p., 492p.

Oliveira, E. G. de \& R. A. Thomazzielo. 1991. A cigarra é uma real ameaça ao seu cafezal. Previna-se. Inf. Téc., CAT1, 4p.
Ruflinelli, A. 1970. Contribucion ai conocimiento de los Homopteros Auquenorrimcos de! Uruguay. Publ. Téc. Ser. Zoo). Agríc.1:1-25.

Schiottfeldt, C. S. 1944. Insetos encontrados em plantas cultivadas e comuns - Viçosa, Minas Gerais. Rev. Ceres 6: 52-65,

Silva, A. G.D.A., C.R. Gonçalves, D.M. Galvão, A. J. L. Gonçalves, J. Gomes, M. do N. Silva \& L. de Simoni. 1968. Quarto catálogo dos insetos que vivem nas plantas do Brasil, seus parasites e predadores.Rio de Janeiro, Min. de Agri. Depto. de Def. e Inspeção Agropecuária, 622 .

Souza, J.C., P.R. Reis \& C.C.A. Melles. 1983. Cigarras do cafeeiro - Histórico, reconhecimento, biologia, prejuízos e controle. EPAMIG, Bol. Téc. $n^{\circ}$ 5, 27p.

Torres, B. A. 1945. Sobre algumas espécies de cicadidos presentes en nuestro pais y citados como prejudiciales a Ia agricultura. Publ. A; Instituto de Sanidad Vegetal 1: 3-10.

Recebido em 22/02/96. Aceito em 17/02/97. 